\title{
Overview and applicability of the analytical platform
}

\author{
W. Raskob, S. Möhrle, S. Bai and T. Müller \\ KIT - Karlsruhe Institute of Technology, Hermann-von-Helmholtz-Platz 1, 76344 Eggenstein-Leopoldshafen, Germany.
}

\begin{abstract}
The European project PREPARE (innovative integrated tools and platforms for radiological emergency preparedness and post-accident response in Europe) aims at closing gaps that have been identified in nuclear and radiological preparedness following the first evaluation of the Fukushima disaster. Among others, a work package was established to develop a so-called analytical platform (AP) exploring the scientific and operational means to improve information collection, information exchange and the evaluation of such types of disasters. The AP contains several modules supporting the work of experts in analysing an ongoing event and in communicating with the public.
\end{abstract}

Keywords: analytical platform / decision support / event analysis / communication / PREPARE

\section{Introduction}

Following Fukushima, many organisations in Europe have set up working groups trying to analyse the event and to predict its future development. In most cases, these working groups had no clearly defined workflow but were driven from requests from mass media, politicians and the public. Several times, the working groups had different input data for their assessments leading of course to different conclusions. Decisions taken by foreign countries aiming at protecting their citizens in Japan were based on these expert assessments resulting in diverging actions e.g. in evacuation of foreign citizens in Japan, iodine prophylaxis in Japan and monitoring of their citizens returning home from Japan. This of course created confusion among the public. Europe as the array of more than 30 countries with various cultural backgrounds and differences in administrative culture needs a common information source where national experts could find information about the event and input data for their assessments.

The European project PREPARE (innovative integrated tools and platforms for radiological emergency preparedness and post-accident response in Europe) aimed at closing these gaps that have been identified in nuclear and radiological preparedness following the first evaluation of the Fukushima disaster. Among others, a work package was established to develop a so-called analytical platform (AP) exploring the scientific and operational means to improve information collection, information exchange and the evaluation of such types of disasters.

\section{Analytical platform (AP)}

The AP provides a framework as it has been designed and developed with the purpose to allow analytical discussion between experts at expert level and to widespread congruent information on the current situation to the public community.
Furthermore, crowd-sourcing facilities should be used to quickly adapt to the development of the situation and to analyse and respond to the arising questions of the public.

The AP is composed of 3 types of tools (see Figure 1):

- tools for facilitating expert-to-expert interactions, including:

- a case-based reasoning tool that comprises machinelearning algorithms to find solutions for events that are not part of the existing knowledge database;

- a multi-criteria analysis tool for evaluating the effects of potential sets of measures to be taken;

- communication means for experts to analyse an on-going event (virtual meeting room);

- a web-crawling tool allowing the collection and processing of information from all possible sources;

- an 'ask-the-expert' tool aiming to communicate to the public about assessments and the future evolution of the event and answering questions from the public.

Details of the AP will be further described in five papers in this special issue, dealing with the technical realisation of the AP, the knowledge database, ask-the-expert and web crawling, the operational procedures of the AP and finally the conditions and means for a useful and trustworthy engagement of experts in the PREPARE analytic platform - survey and interviews outcomes.

\section{Application}

The AP is designed to be centrally installed, and access is provided via a web browser from any mobile or stationary computer. This allows all registered users to access the platform with their access rights and contribute to tasks they have subscribed for. Typically, in the beginning, a task group 


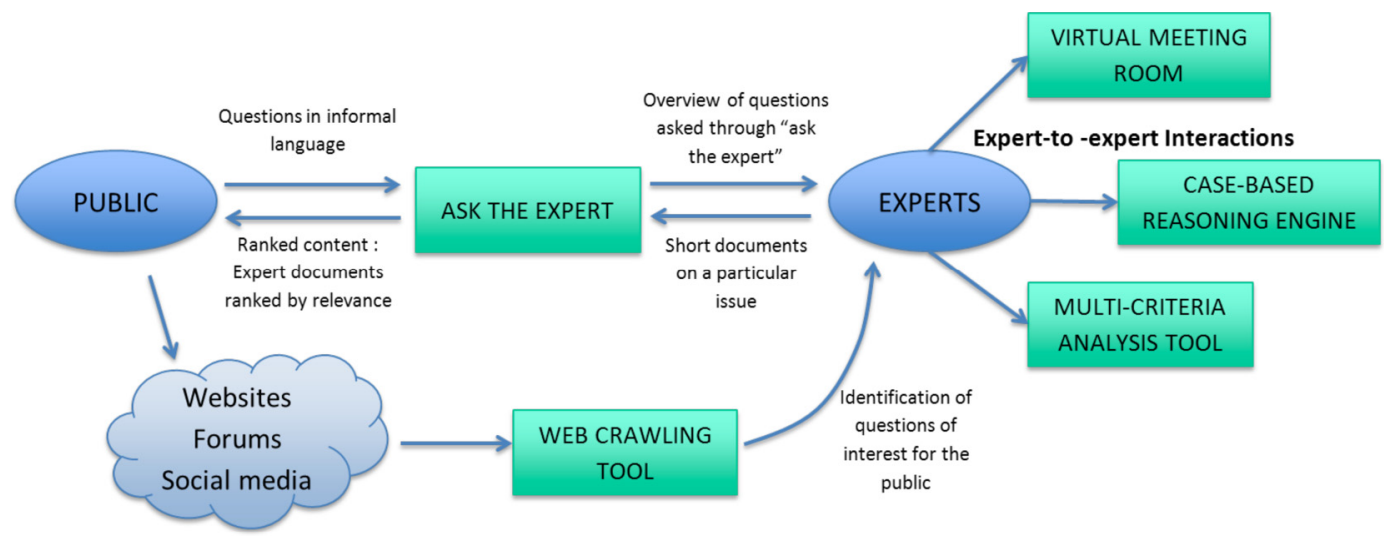

Figure 1. Interactions with the public and the experts through the PREPARE AP tools.

for estimating the source term, evaluating the ongoing event, collecting the necessary information from public available sources and a decision-making team will be established. Depending on the requests and the ongoing emergency, further teams can be initiated.

Key objectives are collecting, analysing and disseminating of information to all relevant stakeholders. Important to note here is the fact that no information will be published without the agreement of the platform operators. In this respect, consensus must be reached, and only commonly agreed information will be disseminated to the public.

The AP was designed to be applicable in all phases of an emergency. In the pre-release phase, the so-called HERCAWENRA scheme (HERCA and WENRA, 2014) was implemented allowing to estimate possible consequences of an nuclear accident based on only very limited information about the plant status and the weather conditions. During and after the release, the knowledge database contains so far more than 100 scenarios and history cases for early phase countermeasures and some tens for the late phase. They can be used as starting point for the evaluation of an on-going event. Of particular importance is the fact that even with very limited information, the knowledge database can provide valuable information on the potential consequences.

Communication internally is supported by the virtual meeting room that allows experts to communicate about particular topics. This communication is secure and only visible to those who have been assigned to that task. The forum and the web crawling facilities support the communication to the public.

Application areas of the AP are also situations where information is sparse and uncertain which might be the case if the accident has happened in a neighbouring country and information flow is limited. Training of decision makers and other experts is also a possible field of application as the AP contains a lot of information for many different scenarios and provides such a vast knowledge on historic consequences but also on particular events from the scenario database.

\section{Discussion and conclusions}

The AP is a unique system that supports experts from all areas in analysing an on-going nuclear or radiological emergency. It serves as a focal point for collecting information, providing means to interpret them and finally to disseminate them to the public.

At the end of the PREPARE project, the AP has been released in its first operational version. The next steps will be to explore their application. In particular, the question of the users has to be discussed. So far, the AP will be managed by the NERIS platform. The NERIS platform represents more than 50 organisations from the operational community, research and Non Governmental Organisations (NGOs), interested in emergency management and long-term rehabilitation. A working group on information, participation and communication has been established aiming to explore the usage of the AP by institutional and non-institutional experts. Also, the connection of official European systems such as EURDEP and ECURIE should be explored.

At present, the AP is maintained by KIT. KIT will provide access to the platform to all interested parties in 2016. Even designed for nuclear events, the AP contains a database for earthquakes. In the near future, additional databases for natural disasters such as floods and thunderstorms will be added. In addition, the scenario database will be further increased by adding more scenarios for inhabited areas and food production systems.

Acknowledgement. The research leading to these results has received funding from the European Atomic Energy Community Seventh Framework Programme FP7/2012-?2013 under grant agreement 323287.

\section{Reference}

HERCA, WENRA (2014) HERCA-WENRA Approach for a Better Cross-Border Coordination of Protective Actions during the Early Phase of a Nuclear Accident. Stockholm. http://www.herca.org/ docstats/HERCA-WENRA\%20approach\%20for\%20better\% 20cross-border\%20coordination.pdf.

Cite this article as: W. Raskob, S. Möhrle, S. Bai, T. Müller. Overview and applicability of the analytical platform. Radioprotection 51 (HS2), S179-S180 (2016). 